Note

\title{
Coherent backscattering: Conceptions and misconceptions (reply to comments by Bruce W. Hapke and Robert M. Nelson)
}

\author{
Victor P. Tishkovets ${ }^{\text {a }}$, Michael I. Mishchenko ${ }^{\text {b,* }}$ \\ a Institute of Radio Astronomy of NASU, 4 Chervonopraporna St., Kharkiv 61002, Ukraine \\ ${ }^{\mathrm{b}}$ NASA Goddard Institute for Space Studies, 2880 Broadway, New York, NY 10025, USA
}

\section{A R T I C L E I N F O}

\section{Article history:}

Received 2 November 2009

Accepted 3 November 2009

\section{Keywords:}

Electromagnetic scattering

Remote sensing

Planetary surfaces

Coherent backscattering

Radiative transfer

\begin{abstract}
A B S T R A C T
Although the note by Hapke and Nelson has virtually no relevance to our original publication, it contains a number of statements that are misleading and/or wrong. We, therefore, use this opportunity to dispel several profound misconceptions that continue to hinder the progress in remote sensing of planetary surfaces.
\end{abstract}

Published by Elsevier Ltd.

\section{Introduction}

In a recent paper [1], we proposed an approximate technique for the calculation of coherent backscattering (CB) by a semi-infinite, plane-parallel discrete random medium (DRM). In their comment [2], Hapke and Nelson (hereafter designated $\mathrm{HN}$ ) picked on one sentence in our paper as an excuse for submitting a note intended to draw attention to a number of papers of theirs that, presumably, have been unjustly ignored by the JQSRT community.

The sentence in question reads: "Unfortunately, we have no reliable experimental or theoretical results which would allow us to test the proposed technique for larger particles and other values of the parameters characterizing the scattering medium." A straightforward clarification in response to $\mathrm{HN}$ would be to replace the words "experimental results" with the words "results of controlled laboratory measurements". Indeed, the measurements cited in HN fall in the category of laboratory observations rather than controlled laboratory experi-

\footnotetext{
* Corresponding author. Tel.: +1 2126785590 ; fax: +1 2126785622

E-mail addresses: tishkovets@ri.kharkov.ua (V.P. Tishkovets), mmishchenko@giss.nasa.gov (M.I. Mishchenko).
}

ments. As such they cannot be used to substantiate a physically-based theory. We believe that, normally, this subtlety would be understood by a knowledgeable reader. Unfortunately, however, Ref. [2] contains a number of statements that are misleading and/or wrong. We, therefore, decided to respond to $\mathrm{HN}$ and use this opportunity to dispel several profound misconceptions that continue to hinder the progress in remote sensing of planetary surfaces.

\section{What is coherent backscattering?}

It is imperative to recognize that the interaction of electromagnetic radiation with any discrete random medium is fully described by the macroscopic Maxwell equations (MMEs) supplemented by appropriate boundary conditions [3-5]. Indeed, at each moment in time, the entire scattering object (e.g., a cloud of water droplets or a particulate regolith surface) can be represented by a specific spatial configuration of a number $N$ of discrete finite particles. Each particle is assumed to be sufficiently large so that its atomic structure can be ignored and the particle can be characterized by optical constants appropriate to bulk matter. In electromagnetic terms, the 
presence of a particle means that the optical constants inside the particle volume are different from those of the surrounding host medium. The spatial distribution of the optical constants throughout the medium dictates the corresponding boundary conditions which, along with the radiation condition at infinity [6], make the solution of the MMEs unique.

Let us assume for a minute that we have at our disposal a direct computer solver of the MMEs (in the form of a suitable \{computer; computer program\} combination) capable of handling electromagnetic scattering by an arbitrarily complex DRM. Then we would not need any other theoretical concept for the interpretation of laboratory or remote-sensing measurements of electromagnetic scattering. Indeed, the output of any measurement could then be modeled by solving the MMEs for a representative set of realizations of a DRM followed by statistical averaging of the relevant optical observables.

Unfortunately, such a solver of the MMEs does not exist and is unlikely to become available in the near future. In practice, one has to simplify the problem by making one or more specific assumptions. The result of these simplifications is twofold. First of all, the problem becomes solvable. Second of all, it becomes both possible and convenient to identify certain idealized theoretical concepts, such as, for example, radiative transfer (RT) and $\mathrm{CB}$. Such concepts are unnecessary in principle and have restricted validity. However, they constitute what is usually called "physical understanding of the problem" and as such may have some positive heuristic value and increase the level of mental comfort of researchers analyzing digital computer outputs or experimental data.

The specific way to introduce the concepts of RT and $\mathrm{CB}$ is to derive the exact Foldy-Lax equations (FLEs) for a multi-particle group from the macroscopic Maxwell equations $[4,7,8]$ and then convert the FLEs into an infinite order-of-scattering expansion [4,5]. In the framework of this formalism, each particle is uniquely and completely characterized by the respective dyadic transition operator. In many cases of practical interest, the original FLEs are intractable and have to be simplified. One of such simplifications is the far-field version of the FLEs wherein it is assumed that each particle is located in the far-field zones of all the other particles forming the group $[4,5,9,10]$. Three fundamental ingredients of the farfield FLEs are as follows:

(i) the partial field scattered by any particle $i$ and exciting any particle $j$ evolves into an outgoing spherical wavelet by the time it reaches particle $j$;

(ii) each particle is now characterized by the scattering dyadic rather than by the dyadic transition operator; and

(iii) instead of the original system of volume integral equations one deals with a system of algebraic equations.

Each partial spherical wavelet is a transverse electromagnetic field and as such is characterized by a phase. This means that one can consider a multiple-scattering wave trajectory and evaluate its phase accumulated by the time it reaches the observation point. Furthermore, one can evaluate the result of the interference of two multiply-scattered waves at the observation point depending on the phase difference between the waves. This ultimately leads to the consideration of so-called ladder and cyclical diagrams [11] and to the derivation of the microphysical vector theories of RT and CB [4,5]. The vector RT theory is essentially the result of summing up self-interference results (i.e., the ladder diagrams) [12,13], wherein a multiply-scattered wave interferes constructively with itself. The effect of $\mathrm{CB}$ is described by the sum of all cyclical (or maximally crossed) diagrams [4,9,11,1421].

It is worth reiterating that both RT and CB are idealized theoretical concepts. Since they follow directly from the Maxwell equations as an outcome of making a sequence of well-defined assumptions [4,5], the practical applicability of the theories of RT and CB does not require any validation provided that all these assumptions are fulfilled. For example, there is little doubt that the RT equation describes adequately the results of photopolarimetric observations of clouds in planetary atmospheres, as exemplified spectacularly by the discovery of micrometer-sized sulfuric acid droplets in the atmosphere of Venus [22]. However, if the RT and CB theories are used to model situations in which one or more of the underlying assumptions are violated then the quantitative applicability of these theories must be carefully examined. The requisite benchmark test results can be obtained either by performing a controlled laboratory experiment or by directly solving the MMEs.

\section{Controlled laboratory experiments versus laboratory observations}

One of the fundamental assumptions that one has to make in order to arrive at the concepts of RT and CB is that the particles forming a DRM are widely separated. However, both concepts have been used frequently to describe the scattering properties of densely packed DRMs such as particle suspensions and particulate surfaces with volume packing densities comparable to or even exceeding $10 \%$. One way to validate the quantitative applicability of the concepts of RT and CB to such DRMs is to compare the results of numerical computations with those of controlled laboratory experiments.

There is a fundamental difference between controlled laboratory experiments and laboratory observations. In the former, one fully controls all the conditions of the experiment and has complete knowledge of all physical parameters specifying the scattering medium (e.g., the size distribution, shape, refractive index, and packing density of the particles and the geometrical dimensions of the scattering medium) as well as the ability to change them one at a time. The known parameters of the medium then serve as input for numerical computations, thereby making possible an unambiguous comparison of theoretical and experimental results. Laboratory observations do not differ from remote-sensing (e.g., astronomical) observations in that one measures only the parameters of 
the scattered light but does not provide independently a complete physical and compositional specification of the scattering medium. As a consequence, laboratory observations (such as those referenced in $\mathrm{HN}$ ) cannot be used for validation purposes.

Instructive examples of the use of controlled laboratory experiments for validating the applicability of the low-packing-density concepts of RT and CB to densely packed DRMs can be found, e.g., in Refs. [1,23,24].

In some rare cases, laboratory observations result in a forceful challenge of an approximate theory of electromagnetic scattering and thereby facilitate the development of an improved approximation. A classical example is the laboratory observation by Lyot [25] of a supernarrow negative-polarization minimum at backscattering angles for a particulate magnesia sample which was later interpreted as the polarization opposition effect (POE) caused by CB [26,27]. The simultaneous laboratory observation of the POE and an equally narrow brightness opposition effect (BOE) for a similar magnesia sample [28] has provided additional support for this interpretation. (It is worth noting that contrary to the assertions in $\mathrm{HN}$, the laboratory data reported in Ref. [28] reveal a pronounced dependence of the opposition intensity peak on the physical parameters of the scattering medium.)

\section{Numerically exact solutions of the Maxwell equations}

Another way to test the validity of idealized theoretical concepts and the quantitative applicability of approximate theories of electromagnetic scattering by DRMs is to use the results of direct computer solutions of the MMEs. There are three numerically exact computer solvers of the MMEs that have been applied recently to model electromagnetic scattering by media consisting of large numbers of randomly positioned particles: the superposition $T$ matrix method [29,30], the discrete dipole approximation [31,32], and the finite-difference time-domain method $[33,34]$. By directly solving the MMEs one can

(i) eliminate any uncertainty associated with the use of an approximate theoretical approach;

(ii) control precisely all physical parameters of the scattering medium and vary them one at a time; and (iii) compute all relevant optical observables at once.

Such modeling can be viewed in many respects as an ideal controlled laboratory experiment in which one can study unambiguously the onset, evolution, and potential decay of all manifestations of RT and CB as the particle packing density gradually increases from zero to values typical of actual particulate surfaces and particle suspensions. Numerous examples of using this approach can be found in Refs. [35-49]. An important result of these studies $[48,49]$ is that all anticipated manifestations of CB, at least for nonabsorbing or weakly absorbing DRMs, are remarkably immune to packing density effects [50-54] and can be observed for volume densities exceeding $30 \%$.

There is no doubt that the ever-increasing efficiency of computers will enable one to explore progressively sophisticated scattering models and will eventually provide the ultimate theoretical tool for the interpretation of remote-sensing observations, thereby rendering the idealized concepts of CB and RT unnecessary.

\section{Use of laboratory observations to test unphysical models}

The Hapke bi-directional reflectance model (which is now claimed to incorporate the effect of $\mathrm{CB}$ ) is an instructive example of an unphysical approach to describe electromagnetic scattering by a DRM. This model does not follow from the MMEs and is an artificial conglomerate of unsubstantiated yet enticingly simple formulas intended to provide a back-of-an-envelope solution of a profoundly complex scattering problem. Since the Hapke model avoids the use of physical parameters involved in the solution of the MMEs and artificially ignores the vector nature of light, it cannot be validated against the results of controlled laboratory experiments. Instead, the traditional way to "validate" the Hapke model has been to compare its predictions with results of laboratory observations, the closeness of the resulting fit being the only success criterion. As a result, it has been established that the Hapke model is a remarkable interpolation tool capable of fitting virtually any observation results. Unfortunately, the price one has to pay for this universal interpolation capability is that the best-fit model usually has little or no physical meaning [55]. As discussed in [56], examples of such unphysical results are the "discovery" of isotropically scattering cloud particles in the atmosphere of Venus, the "discovery" of backscattering particles with negative asymmetry parameters, and the violation of the energy conservation law.

The way in which the Hapke model is usually applied to astrophysical observations and yields "definitive" results is equally suspect. Indeed, spatially integrated telescopic observations of a heterogeneous planetary surface yield a complex convolution of contributions from morphologically different surface types with varying albedos. Some of them can cause BOE and POE of varying angular widths and amplitudes, and some of them can cause only the more robust but still spatially varying BOE. Therefore, it is highly problematic, if even possible, to model such data unambiguously, unless one uses a synthetic interpolation function capable of fitting almost any data at the likely expense of being physically meaningless.

\section{Use of laboratory observations to interpret remote- sensing observations}

Another misconception pursued in the publications by Hapke et al. cited in Ref. [2], is that laboratory observations can be used to interpret remote-sensing observations. The way this is done is to (i) postulate that what is observed in the laboratory is the effect of $\mathrm{CB}$; (ii) claim that the results of remote-sensing observations are wellrepresented by the results of laboratory observations; and 
(iii) conclude that what is observed remotely is also the effect of $\mathrm{CB}$.

The fallaciousness of this approach is obvious. As we have already emphasized, CB is an idealized theoretical concept. Therefore, the only way to establish the $\mathrm{CB}$ nature of a laboratory or remote-sensing observation is to compare the measurement results with the results of theoretical computations.

A good illustration of this profound misconception is the alleged result by Hapke et al. that $C B$ always serves to enhance the circular polarization ratio and suppress the linear polarization ratio. This result appears to have been derived from their laboratory observations, is claimed to be an unequivocal experimental identifier of the effect of $C B$, and has allegedly been used to prove the substantial presence of $\mathrm{CB}$ in the sunlight reflected by the lunar surface. However, direct computer solutions of the MMEs reported in Refs. $[38,46,48,49]$ demonstrate that, in general, this result is wrong and that CB can either enhance or suppress the linear polarization ratio depending on the physical parameters of a specific DRM.

Another byproduct of this misconception can be found in HN. Indeed, the second paragraph contains a claim that laboratory observations referenced in HN show unequivocally the effect of $\mathrm{CB}$, whereas the last paragraph claims that we do not actually know what $C B$ is.

In fact, exactly the opposite is true: we know precisely what $\mathrm{CB}$ is, but do not necessarily know what was observed in the laboratory by Hapke et al.

\section{Concluding remarks}

Like any idealized and approximate theoretical concept, the concepts of RT and CB have their limitations and, in the final analysis, are unnecessary. The range of quantitative applicability of these concepts in situations violating the basic assumptions listed in Refs. [4,5] needs to be established by using the results of controlled laboratory experiments and/or numerically exact computer solutions of the MMEs. Furthermore, because of the purely theoretical nature of these concepts, their relevance to optical effects observed remotely or in the laboratory for particulate suspensions and surfaces can be established only by comparing the results of observations with physically-based theoretical results $[48,57]$.

It is imperative to recognize that the limited validity of the inherently approximate concepts of RT and $\mathrm{CB}$ does not invalidate the MMEs. It is, therefore, preposterous to suggest (see the final paragraph of HN) that the laboratory observations by Hapke and Nelson make necessary as major a development in physics as the discovery of the analog of Planck's law for CB.

\section{Acknowledgment}

This project was sponsored by the NASA Radiation Sciences Program managed by Hal Maring.

\section{References}

[1] Tishkovets VP, Mishchenko MI. Approximate calculation of coherent backscattering for semi-infinite discrete random media. J Quant Spectrosc Radiat Transfer 2009;110:139-45.

[2] Hapke BW, Nelson RM. Comments on "Approximate calculation of coherent backscattering for semi-infinite discrete random media" by Victor P. Tishkovets and Michael I. Mishchenko. J Quant Spectrosc Radiat Transfer 2010;111:659-60.

[3] Stratton JA. Electromagnetic theory. New York: McGraw-Hill; 1941.

[4] Mishchenko MI, Travis LD, Lacis AA. Multiple scattering of light by particles: radiative transfer and coherent backscattering. Cambridge, UK: Cambridge University Press; 2006.

[5] Mishchenko MI. Multiple scattering, radiative transfer, and weak localization in discrete random media: unified microphysical approach. Rev Geophys 2008;46:RG2003.

[6] Müller C. Foundations of the mathematical theory of electromagnetic waves. Berlin: Springer; 1969.

[7] Tsang L, Kong JA, Shin RT. Theory of microwave remote sensing. New York: Wiley; 1985.

[8] Babenko VA, Astafyeva LG, Kuzmin VN. Electromagnetic scattering in disperse media: inhomogeneous and anisotropic particles. Chichester, UK: Praxis; 2003.

[9] Tishkovets VP. Multiple scattering of light by a layer of discrete random medium: backscattering. J Quant Spectrosc Radiat Transfer 2002;72:123-37.

[10] Tishkovets VP, Mishchenko MI. Coherent backscattering of light by a layer of discrete random medium. J Quant Spectrosc Radiat Transfer 2004;86:161-80.

[11] Barabanenkov YuN, Kravtsov YuA, Ozrin VD, Saichev AI. Enhanced backscattering in optics. Progr Opt 1991;29:65-197.

[12] Mishchenko MI. Vector radiative transfer equation for arbitrarily shaped and arbitrarily oriented particles: a microphysical derivations from statistical electromagnetics. Appl Opt 2002;41:7114-34.

[13] Mishchenko MI. Microphysical approach to polarized radiative transfer: extension to the case of an external observation point. Appl Opt 2003;42:4963-7.

[14] Lagendijk A, van Tiggelen BA. Resonant multiple scattering of light. Phys Rep 1996;270:143-215.

[15] Kuz'min VL, Romanov VP. Coherent phenomena in light scattering from disordered systems. Phys-Uspekhi 1996;39:231-60.

[16] van Rossum MCW, Nieuwenhuizen ThM. Multiple scattering of classical waves: microscopy, mesoscopy, and diffusion. Rev Mod Phys 1999;71:13-371.

[17] Lenke R, Maret G. Multiple scattering of light: coherent backscattering and transmission. In: Brown W, Mortensen K, editors Scattering in polymeric and colloidal systems. Amsterdam: Gordon and Breach; 2000. p. 1-73.

[18] Tishkovets VP, Litvinov PV, Lyubchenko MV. Coherent opposition effect for semi-infinite discrete random medium in the doublescattering approximation. J Quant Spectrosc Radiat Transfer 2002;72:803-11.

[19] Tishkovets VP, Mishchenko MI. Coherent backscattering of light by a layer of discrete random medium. J Quant Spectrosc Radiat Transfer 2004;86:161-80.

[20] Muinonen K. Coherent backscattering of light by complex random media of spherical scatterers: numerical solution. Waves Random Media 2004; 14:365-88.

[21] Litvinov P, Tishkovets V, Ziegler K. Coherent backscattering effects for discrete random media. J Quant Spectrosc Radiat Transfer 2006;103:131-45.

[22] Hansen JE, Hovenier JW. Interpretation of the polarization of Venus. J Atmos Sci 1974;31:1137-60.

[23] Wolf P, Maret G. Weak localization and coherent backscattering of photons in disordered media. Phys Rev Lett 1985;55:2696-9.

[24] Mishchenko MI. Polarization effects in weak localization of light: calculation of the copolarized and depolarized backscattering enhancement factors. Phys Rev B 1991;44:12597-600.

[25] Lyot B. Recherches sur la polarisation de la lumière des planetes et de quelques substances terrestres. Ann Observ Paris Sect Meudon 1929;8:1.

[26] Mishchenko MI. On the nature of the polarization opposition effect exhibited by Saturn's rings. Astrophys J 1993;411:351-61.

[27] Mishchenko MI, Luck J-M, Nieuwenhuizen TM. Full angular profile of the coherent polarization opposition effect. J Opt Soc Am A 2000;17:888-91.

[28] Shkuratov Yu, Ovcharenko A, Zubko, E, et al. The opposition effect and negative polarization of structural analogs for planetary regoliths. Icarus 2002;159:396-416. 
[29] Mackowski DW, Mishchenko MI. Calculation of the $T$ matrix and the scattering matrix for ensembles of spheres. J Opt Soc Am A 1996;13:2266-78.

[30] Mishchenko MI, Travis LD, Lacis AA. Scattering, absorption, and emission of light by small particles. Cambridge: Cambridge University Press; 2002. Available in the PDF format at <http:// www.giss.nasa.gov/staff/mmishchenko/publications/ $>$.

[31] Draine BT. The discrete dipole approximation for light scattering by irregular targets. In: Mishchenko MI, Hovenier JW, Travis LD, editors. Light scattering by nonspherical particles: theory, measurements, and applications. San Diego: Academic Press; 2000. p. 131-45.

[32] Yurkin MA, Hoekstra AG. The discrete dipole approximation: an overview and recent developments. J Quant Spectrosc Radiat Transfer 2007;106:558-89.

[33] Yang P, Liou KN. Finite difference time domain method for light scattering by nonspherical and inhomogeneous particles. In: Mishchenko MI, Hovenier JW, Travis LD, editors. Light scattering by nonspherical particles: theory, measurements, and applications. San Diego: Academic Press; 2000. p. 173-221.

[34] Taflove A, Hagness SC. Computational electrodynamics: the finitedifference time-domain method. Boston: Artech House; 2000.

[35] Tseng, SH, et al. Simulation of enhanced backscattering of light by numerically solving Maxwell's equations without heuristic approximations. Opt Express 2005;13:3666-72.

[36] Tseng, SH, et al. Pseudospectral time domain simulations of multiple light scattering in three-dimensional macroscopic random media. Radio Sci 2006;41:RS4009.

[37] Mishchenko MI, Liu L. Weak localization of electromagnetic waves by densely packed many-particle groups: exact 3D results. J Quant Spectrosc Radiat Transfer 2007;106:616-21.

[38] Mishchenko MI, Liu L, Mackowski, DW, et al. Multiple scattering by random particulate media: exact 3D results. Opt Express 2007;15: 2822-2836.

[39] Mishchenko MI, Liu L, Videen G. Conditions of applicability of the single-scattering approximation. Opt Express 2007;15: $7522-7527$

[40] Mishchenko MI, Liu L, Hovenier JW. Effects of absorption on multiple scattering by random particulate media: exact results. Opt Express 2007;15:13182-7.

[41] Tseng SH, Huang B. Comparing Monte Carlo simulation and pseudospectral time-domain numerical solutions of Maxwell's equations of light scattering by a macroscopic random medium. Appl Phys Lett 2007;91:051114.

[42] Mackowski DW, Mishchenko MI. Prediction of thermal emission and exchange among neighboring wavelength-sized spheres. J Heat Transfer 2008;130:112702.
[43] Tseng SH. Optical characteristics of a cluster of closely-packed dielectric spheres. Opt Commun 2008;281:1986-90.

[44] Sukhov A, Haefner D, Dogariu A. Coupled dipole method for modeling optical properties of large-scale random media. Phys Rev E 2008;77:066709.

[45] Okada Y, Kokhanovsky AA. Light scattering and absorption by densely packed groups of spherical particles. J Quant Spectrosc Radiat Transfer 2009;110:902-17.

[46] Mishchenko MI, Liu L. Electromagnetic scattering by densely packed particulate ice at radar wavelengths: exact theoretical results and remote-sensing implications. Appl Opt 2009;48: 2421-2426.

[47] Voit F, Schäfer J, Kienle A. Light scattering by multiple spheres: comparison between Maxwell theory and radiative-transfer-theory calculations. Opt Lett 2009;34:2593-5.

[48] Mishchenko MI, Dlugach JM, Liu, L, et al. Direct solutions of the Maxwell equations explain opposition phenomena observed for high-albedo solar system objects. Astrophys J 2009;705:L118-22.

[49] Mishchenko MI, Dlugach JM, Liu L. Azimuthal asymmetry of the coherent backscattering cone: exact theoretical results. Phys Rev A [in press].

[50] Tishkovets V, Shkuratov Yu, Litvinov P. Comparison of collective effects at scattering by randomly oriented clusters of spherical particles. J Quant Spectrosc Radiat Transfer 1999;61:767-73.

[51] Tishkovets V, Petrova E, Jockers K. Optical properties of aggregate particles comparable in size to the wavelength. J Quant Spectrosc Radiat Transfer 2004;86:241-65.

[52] Petrova E, Tishkovets V, Jockers K. Modeling of opposition effects with ensembles of clusters: interplay of various scattering mechanisms. Icarus 2007;188:233-45.

[53] Tishkovets VP. Light scattering by closely packed clusters: shielding of particles by each other in the near field. J Quant Spectrosc Radiat Transfer 2008;109:2665-72.

[54] Tishkovets VP. Incoherent and coherent backscattering of light by a layer of densely packed random medium. J Quant Spectrosc Radiat Transfer 2007;108:454-63.

[55] Mishchenko MI. Asymmetry parameters of the phase function for densely packed scattering grains. J Quant Spectrosc Radiat Transfer 1994:52:95-110.

[56] Mishchenko MI, Macke A. Asymmetry parameters of the phase function for isolated and densely packed spherical particles with multiple internal inclusions in the geometric optics limit. J Quant Spectrosc Radiat Transfer 1997;57:767-94.

[57] Mishchenko MI, Rosenbush VK, Kiselev NN. Weak localization of electromagnetic waves and opposition phenomena exhibited by high-albedo atmosphereless solar system objects. Appl Opt 2006;45:4459-63. 